\title{
Top 20 translational researchers of 2015
}

\author{
Brady Huggett \& Kathryn Paisner
}

Our annual ranking of faculty (Table 1) on the basis of patent grants research from IP Checkups and publications (via $\mathrm{H}$ index) shows many of the same faces as 2014. New entrants include investigators from the Mainz University Medical Center, Osaka University, Harvard College, California Institute of Technology, University of

Southern California, Icahn School of Medicine at Mount Sinai, the Sloan Kettering Institute and the University of Pennsylvania. Table 2 lists the most-cited patents overall for 2011-2015. In Figure 1, peptide/ protein therapy has surpassed genotyping/sequencing as the category with the most-cited patents.

\section{Table 1 Top 20 translational researchers in 2015}

\section{Inventor/first assignee}

Carlo M. Croce/Ohio State University; Thomas Jefferson University

George A. Calin/Ohio State University; Thomas Jefferson University

Richard D. DiMarchi/Indiana University; Istituto di Ricerche di Biologia Molecolare P. Angeletti University of Mainz; Ganymed

Robert S. Langer/Massachusetts Institute of Technology; Children's

Medical Center Corp.; Harvard College; Brigham \& Women's Hospital;

Technische Universität München; National Tsing Hua University; Dynamic Biosensors

Haruo Sugiyama/International Institute of Cancer Immunology; Osaka University

James M. Wilson/University of Pennsylvania

Patents

granted 2015 Number and title of most-cited patent for 2011-2015 (no. of citations, as of 8/22/2016) H index US 8,084,199 Method of diagnosing poor survival prognosis colon cancer using microRNA-21 (12) 200

20 US 8,389,210 MicroRNA expression abnormalities in pancreatic endocrine and acinar tumors (4) 91

17 US 8,507,428 Glucagon analogs exhibiting GIP receptor activity (5)

48

17 and 16 US 8,168,427 Monoclonal antibodies against claudin-18 for treatment of cancer (6) 47 and

16 US 8,450,298 Amino alcohol lipidoids and uses thereof (49)

David R. Liu/Harvard College; Scripps Research Institute; University of California

Peter G. Schultz/Scripps Research Institute; University of California; IRM LLC Shinya Yamanaka/Kyoto University; National Institute of Advanced Industrial Science and Technology (Japan); Japan Biological Informatics Consortium Karl Deisseroth/Stanford University

Thomas H. Tuschl/University of Massachusetts; Max Planck Institute; Whitehead Institute; M.I.T.; Rockefeller University

Feng Zhang/Broad Institute; M.I.T.; Harvard; Stanford

Michael Koslowski/University of Mainz; Ganymed Pharmaceuticals

Axel Scherer/CalTech; University of Southern California; Boeing; APIC Corp.

Peter Palese and Adolfo Garcia-Sastre/lcahn School of Medicine at Mount Sinai; St. Jude's; Sloan Kettering Institute

David B. Weiner/University of Pennsylvania; Inovio Pharmaceuticals

15

12

12 US 7,906,111 Adeno-

12 US 7,998,904 Evolving new molecular function (20) 51

12 US 7,951,592 Compositions and methods for inducing cell dedifferentiation (3) 115

11 US 8,048,999 Nuclear reprogramming factor (59)

10 US 8,398,692 System for optical stimulation of target cells (40) 99

10 US 8,362,231 RNA interference mediating small RNA molecules (11)

10 US 8,697,359 CRISPR-Cas systems and methods for altering expression of gene products (74) 53

10 US 9,216,218; US 8,975,375; US 8,961,980 Monoclonal antibodies for treatment of cancer; 26 dentification of tumor-associated antigens for diagnosis and therapy; Monoclonal antibodies for treatment of cancer (1)

9 US 7,887,753 Apparatus and methods for conducting assays and high throughput screening (19) 74 9 and 9 US 8,591,881 Chimeric Newcastle disease viruses and uses thereof (10) 96 and

9 US 8, 183,352; US 8,178,660 Attenuated vif DNA immunization cassettes for genetic vac- 10 13

(1)
15 69 cines \& vaccines and immunotherapeutics using codon optimized IL-15 and methods for using the same (30)

a $\mathrm{H}$ index is supplied by researcher, pulled from Google Scholar or calculated through Thomson Reuters Web of Science; it is calculated to measure the impact of a scientist's body of published work, the higher the number the greater the impact. Seven researchers tied with eight patents granted in 2015.

Table 2 Most-cited patents, 2011-2015

Patent no. and title

US 8,112,142B2/USRE 43282E1 Assessing the condition of a joint and devising treatment

US 8,298,677B2 Fluorescent silica-based nanoparticles

US 7,932,034B2 Heat and $\mathrm{pH}$ measurement for sequencing of DNA US 7,888,013B2 Method of analyzing DNA sequence using field-effect device, and base sequence analyzer

US 7,888,017B2 Non-invasive fetal genetic screening by digital analysis
Inventor/assignee(s)/applicant(s)

Publication Patent citations, as year

Eugene J. Alexander, Thomas P. Andriacchi, Philipp Lang, Daniel Steines/Stanford 2012 University

Ulrich Weisner, Hooisweng Ow, Daniel R. Larson and Watt W. Webb/Cornell University Hesaam Esfandyarpour and Mostafa Ronaghi/Stanford University

Yuji Miyahara, Toshiya Sakata/National Institute for Materials Science (Japan)

Stephen Quake and Hei-Mun Christina Fan/Stanford University (1) 6

(1)




\section{Erratum: Top 20 translational researchers of 2015}

\section{Brady Huggett \& Kathryn Paisner}

Nat. Biotechnol. doi:10.1038/bioe.2016.9; published online 22 September 2016; corrected online 11 October 2016

In the version of this article initially published, in Table 1, the $\mathrm{H}$ indexes for several authors were incorrect. Author names, correct index (incorrect index): Calin, 91 (69); DiMarchi, 48 (36); Sahin, 47 (6); Langer, 228 (225); Liu, 51 (34); Deisseroth, 99 (98); Zhang, 53 (42); Palese, 96 (49); and Garcia-Sastre, 101 (61). The errors have been corrected in the HTML and PDF versions of the article. 